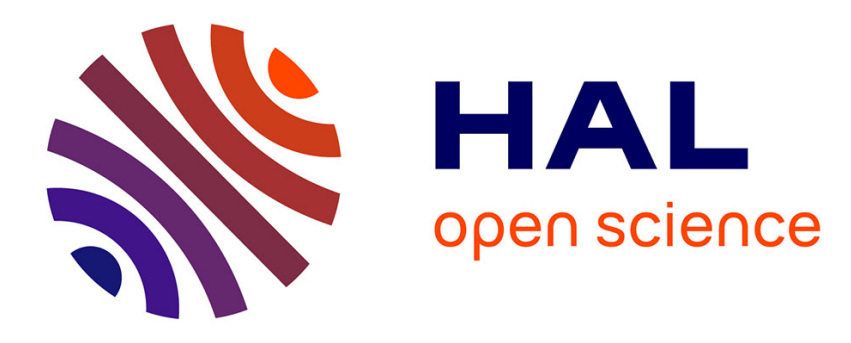

\title{
Quelques réflexions sur les sources de variations d'isolats A 1 de Phytophthora infestans (Mont.) de Bary (*)
}

Françoise Le Grand-Pernot

\section{To cite this version:}

Françoise Le Grand-Pernot. Quelques réflexions sur les sources de variations d'isolats A 1 de Phytophthora infestans (Mont.) de Bary (*). Agronomie, 1986, 6 (4), pp.321-324. hal-00884881

\section{HAL Id: hal-00884881 \\ https://hal.science/hal-00884881}

Submitted on 1 Jan 1986

HAL is a multi-disciplinary open access archive for the deposit and dissemination of scientific research documents, whether they are published or not. The documents may come from teaching and research institutions in France or abroad, or from public or private research centers.
L'archive ouverte pluridisciplinaire HAL, est destinée au dépôt et à la diffusion de documents scientifiques de niveau recherche, publiés ou non, émanant des établissements d'enseignement et de recherche français ou étrangers, des laboratoires publics ou privés. 


\title{
Quelques réflexions sur les sources de variations d'isolats $\mathrm{A}_{1}$ de Phytophthora infestans (Mont.) de Bary \\ (*)
}

\author{
Françoise LE GRAND-PERNOT \\ Université de Paris-Sud, Laboratoire de Cryptogamie, associé au C.N.R.S. - U.A. 86, bâtiment 402, F 91405 \\ Orsay
}

Phytophthora infestans, agent du mildiou de la pomme de terre possède un cycle biologique diploïde. A partir de résultats antérieurs sur la transmission de certains caractères morphologiques et physiologiques à travers la reproduction sexuelle, qui ont montré la nature fortement hétérozygote des souches parentales et récessive des allèles de virulence $1,2,3$ et 4 , nous engageons ici une courte réflexion pour tenter d'analyser les capacités de $P$. infestans de faire face rapidement à une situation nouvelle. Ainsi, dans la nature, $P$. infestans tient en réserve sous la forme hétérozygote des potentialités originales ; des caractères de virulence pourront être emmagasinés dans le génome, sans que l'on puisse connaître provisoirement leur existence. L'origine et le rôle des différentes structures végétatives du champignon (mycélium, sporocystes, zoospores) peuvent favoriser l'expression de nouvelles potentialités, leur dissémination et leur conservation. Mieux, la mise en évidence d'une phase sexuelle chez les lignées $A_{1}$, en présence de n'importe quel partenaire complémentaire du même genre, augmente considérablement l'hétérogénéité des pathotypes et les chances de conserver à long terme une population variée. Ainsi la présence potentielle de races complexes dans la nature rend dangereuse l'introduction de nouvelles variétés de pommes de terre dotées d'une ou plusieurs résistances spécifiques.

Mots clés additionnels : Diploïde, hétérozygote, hétérocaryon, virulence récessive, recombinaison mitotique, reproduction sexuelle.

Additional key words : Diploid, heterozygous, heterocaryon, recessive virulence, mitotic recombination, sexual reproduction.

Phytophthora infestans (Mont.) de Bary, agent du mildiou de la pomme de terre constitue encore un danger malgré la mise en œuvre de méthodes chimi-

$\left(^{*}\right)$ N.B. Ces quelques réflexions s'appuient sur des résultats obtenus au laboratoire et regroupés dans le mémoire de thèse (LE GraND, 1983). Deux articles soumis prochainement à Agronomie présenteront les principaux résultats qui nous ont conduite à cette brève analyse. ques et génétiques de lutte. L'introduction de gènes majeurs de résistance, les gènes « $\mathrm{R}$ », dans le génome Solanum tuberosum, la recherche de nouvelles molécules fongicides, leur application au champ demeurent aléatoires, puisque le parasite semble toujours contourner les difficultés en répondant par la création de nouvelles races virulentes ou résistantes aux produits fongitoxiques.

Chez de nombreux Phytophthora, l'accomplisse- 
ment du cycle sexuel peut expliquer facilement l'obtention de nouveaux pathotypes et leur hétérogénéité dans les populations naturelles. Ce phénomène a été constaté au Mexique, aire d'origine de $P$. infestans, où les 2 types de souches $\mathrm{A}_{1}$ et $\mathrm{A}_{2}$ de ce champignon hétérothallique coexistent (GALLEGLY \& GALINDO, 1958), assurant ainsi par reproduction sexuelle le polymorphisme de l'espèce. Toutefois en Europe et en Amérique du Nord, $P$. infestans n'est présent que sous la forme $A_{1}$, il semble donc légitime d'écarter, dans un premier temps, ce mode de variation et d'envisager hors des frontières mexicaines l'intervention d'autres phénomènes pour rendre compte de la diversité et de la variabilité intraspécifique. Ainsi mutation, hétérocaryose et parasexualité sont invoquées et ont pu être généralement prouvées (GALLEGLY \& EICHENMULLER, 1959 ; WILDE, 1961 ; LEACH \& RICH, 1969 ; DENWARD, 1970 ; MALCOLMSON, 1970).

Chez un organisme haploïde, l'obtention de nouveaux individus par recombinaison mitotique n'est possible que si ce mécanisme intervient entre noyaux végétatifs différents. La présence d'une structure hétérocaryotique pouvant se maintenir facilement favorisera donc le phénomène. En revanche, ce mécanisme chez un diploïde n'implique pas forcément la présence de noyaux différents si l'organisme est naturellement hétérozygote.

Bien que le degré de ploïdie de la phase végétative des représentants hétérothalliques du genre Phytophthora ait suscité de nombreuses controverses, il semble bien établi maintenant que le cycle est diploïde (SATOUR \& BUTLER, 1968 ; BOCCAS, 1971 ; SHAW et al., 1973 ; MAİA et al., 1976 ; LE GRAND-PERNOT \& PELlEGRIN, 1976 et de nombreux autres).

Ainsi les variations génétiques peuvent être stockées. Des caractères récessifs peuvent être conservés à l'état latent et ne s'exprimer qu'à la suite d'une mutation ou d'une recombinaison mitotique, si les isolats sont naturellement hétérozygotes. En outre, la descendance sera d'autant plus diversifiée que le mycélium renfermera plus d'hétérocaryons. Or ces deux situations (hétérozygotie et hétérocaryose) existent chez $P$. infestans.

- SANSOME (1977), a observé que des isolats britanniques étaient naturellement hétérocaryotiques puisque des noyaux diploïdes et polyplö̈des coexistaient dans une même souche. Rappelons que bien d'autres auteurs ont également montré que les populations naturelles de différentes espèces de Phytophthora sont assez souvent faites d'éléments hétérocaryotiques (SHEPHERD \& PRATT, 1974 ; MORTIMER et al., 1977 ; HOBE, 1981 ; ERWIN, 1982). Le mycélium peut donc se conserver, sous cette forme, dans des tubercules de pomme de terre infectés. Lorsque les conditions favorables à la croissance du champignon sont de nouveau réunies, le mycélium se développe et produit très rapidement des sporocystes qui peuvent être disséminés par le vent, la pluie ou le sol. La condition multinucléée et la double origine des noyaux du sporocyste (migration nucléaire puis divisions mitotiques) permettent, d'une part, le maintien et la propagation de la structure hétérocaryotique au cours du cycle infectieux et, d'autre part, favorisent l'apparition, au sein même du sporocyste, de noyaux recombinés, dont l'isolement est rendu possible par fragmen- tation du protoplasme et différenciation en zoospores uninucléées. Cependant, chez un descendant dont la structure hétérocaryotique s'est maintenue près de 3 ans au laboratoire (LE GRAND-PERNOT, 1983), la production de spores se répartit en 2 lots : 66 p. 100 des zoospores sont uninucléées et 34 p. 100 sont binucléées, révélant ainsi que la plus petite particule infectieuse, la zoospore, peut, dans certaines occasions, véhiculer une double information.

- La présence dans la nature de thalles hétérocaryotiques n'est pas seule responsable des possibilités de variations rencontrées. L'analyse de la transmission de caractères aisément observables comme la vitesse de croissance, la sporulation, la vitesse de croissance et la morphologie des souches à $10^{\circ} \mathrm{C}$, ainsi qu'un autre caractère directement utile pour la protection de la pomme de terre, à savoir la réaction de compatibilité ou d'incompatibilité face à une gamme de plantes porteuses de gènes $R_{1}, R_{2}, R_{3}$ ou $R_{4}$, ont permis de déceler des structures hétérozygotes chez les lignées parentales pour tous les caractères étudiés. TOOLEY \& FRY (1983) ont abouti aux mêmes conclusions après avoir observé, pour une vingtaine d'enzymes, les spectres protéiques de 26 isolats naturels de $P$. infestans.

La différenciation des organes sexuels chez les souches de type $A_{1}$, potentiellement hermaphrodites, n'est pas inductible uniquement par des partenaires de type $\mathrm{A}_{2}$ de la même espèce, $P$. infestans. Cette induction de nature hormonale (Ko, 1978) peut être produite par d'autres espèces de Phytophthora : $P$. palmivora, $P$. parasitica, $P$. cinnamomi, $P$. capsici, $P$. nicotianae et bien d'autres (SAVAGE et al., 1968 ; BoCCAS, 1978 ; LE GRAND-PERNOT, 1983). Les produits d'hybridation interspécifiques avortent. En revanche, les produits d'autofécondation de chacun des partenaires sont nombreux et bien formés. Même si la rencontre dans la nature de souches compatibles d'espèces différentes est un phénomène rare, SKIDMORE et al., (1984) ont montré qu'elle était possible, puisqu'ils ont pu isoler $P$. drechsleri de compatibilité $\mathrm{A}_{2}$ à partir de pommes de terre. Aussi le déclenchement de la reproduction sexuelle chez des confrontants appartenant à des espèces différentes joue un rôle important à double titre : il favorise la recombinaison génétique, il est à l'origine de la formation d'organes de conservation. Chez $P$. infestans, qui ne différencie pas de chlamydospores, les oospores subissent obligatoirement une phase de dormance de plusieurs mois avant de germer et de redonner naissance à un thalle ou à un sporocyste. La levée de dormance n'entraîne cependant pas la germination simultanée de toutes les oospores. Nombre d'entre elles peuvent rester en phase de latence et peupler ainsi le sol. Elles assurent une conservation de l'espèce à moyen et long terme qui est d'autant plus efficace que les informations portées par les différents individus qui constituent la population dans le sol sont plus diversifiées. Ainsi les oospores dont le noyau diploïde provient de la fusion de 2 noyaux gamétiques favorisent l'expression, à l'état homozygote, des potentialités mises en réserves sous la forme hétérozygote et, dans ce cas, la probabilité d'obtenir un génotype récessif sera plus élevée. Cette dernière remarque est particulièrement importante car nous avons pu attribuer aux allèles de virulence $1,2,3$ et 4 un caractère récessif et démontrer que, dans la majorité des cas, l'expression de 
nouvelles virulences se manifestait à travers les produits de la reproduction sexuelle mais jamais directement à partir des isolats originels étudiés et que ces mêmes isolats étaient hétérozygotes pour les caractères de virulence démasqués lors des croisements (LE GRAND-PERNOT, 1983). De même, on a démontré que le pouvoir pathogène de $P$. parasitica sur œillet était sous la dépendance de gènes récessifs (MAÏA, comm. pers.).

A l'aide d'un schéma (fig. 1), nous avons résumé les différentes étapes du cycle biologique du champignon et montré l'évolution caryologique possible dans les différentes structures d'un thalle contenant à l'origine un seul type de noyau diploïde dont l'hétérozygotie est matérialisée par la présence d'un couple d'hétéroallèles $A V_{2}$ et $\mathrm{av}_{2}$ (fig. $1 \mathrm{~A}$ ) où $a v_{2}$ représente l'allèle récessif de virulence 2 et $A V_{2}$ l'allèle dominant d'avirulence correspondant. Ainsi, le thalle originel $\frac{\mathrm{AV}_{2}}{\mathrm{av}_{2}}$ n'est pas capable d'attaquer les variétés de pomme de terre dotées du gène de résistance spécifique $R_{2}$; il n'exprime pas la virulence 2 .

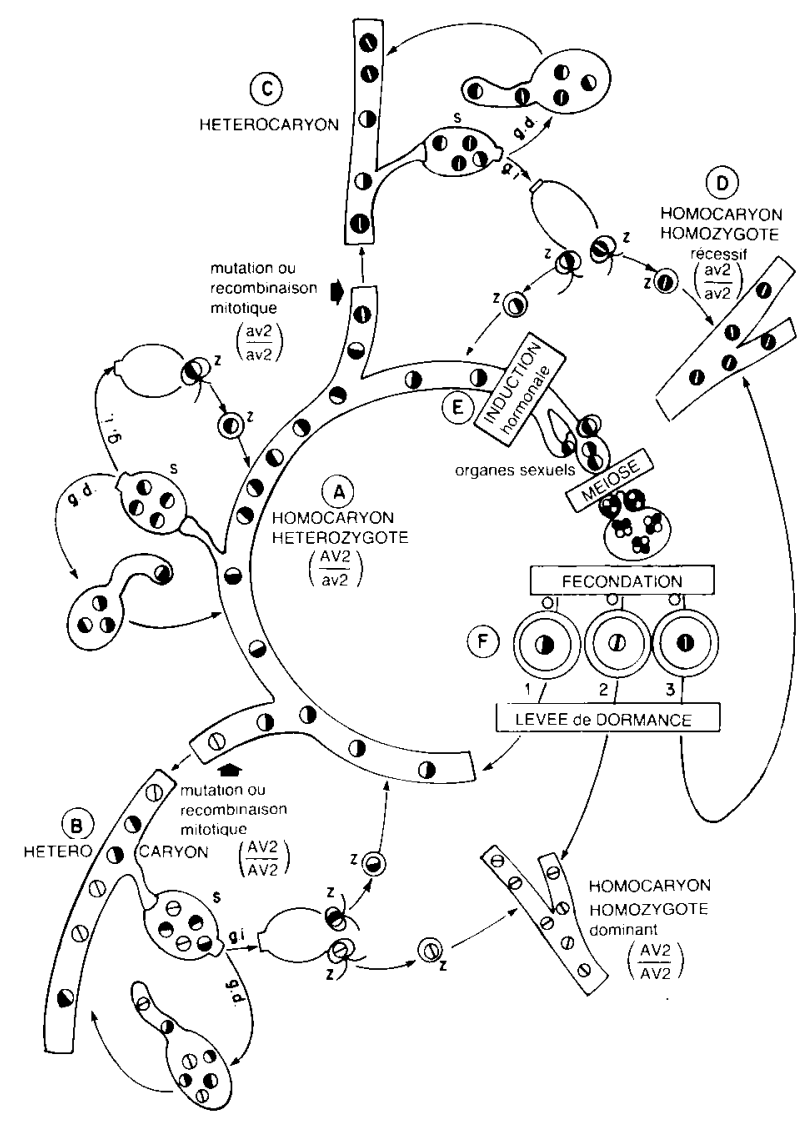

Figure 1

Evolution caryologique d'un homocaryon à noyaux hétérozygotes au cours du cycle biologique de Phytophthora infestans.

Possibility of karyological evolution in a homokaryon with heterozygous nuclei during the biological cycle of Phytophthora infestans.

(s: sporocyste; $z:$ zoospore; g.i. : germination indirecte ; g.d. : germination directe; $o$ : oospore).

Noyaux porteurs de(s) : allèle av $: \bullet ; A V_{2}: 0$; allèles $\frac{a_{2}}{a w_{2}}: \bullet$; $\frac{A V_{2}}{a v_{2}}: \ominus ; \frac{A V_{2}}{11}: \ominus$
Un événement mutationnel, quel que soit le noyau touché, qui interviendra au niveau du locus considéré, pourra modifier la structure du gène vers l'homozygotie dominante $\frac{\mathrm{AV}_{2}}{\mathrm{AV}}$ (fig. 1B) ou récessive (fig. 1C). Si le noyau nouvellement formé a des chances de se maintenir et de se multiplier, l'hyphe deviendra alors hétérocaryotique ( $\mathrm{B}$ ou $\mathrm{C}$ ). Il semble qu'il en soit ainsi dans la nature. Outre la mutation, la recombinaison mitotique entre 2 noyaux $\frac{\mathrm{AV}_{2}}{\mathrm{av} \mathrm{v}_{2}}$ aboutira aux mêmes résultats.

La formation de sporocystes (S) puis de zoospores (Z) peut avoir des conséquences importantes au niveau épidémiologique : la germination directe (g.d.) des sporocystes permettra de maintenir la structure hétérocaryotique de l'hyphe dont ils sont issus (c'est alors la situation exposée au début) ; leur germination indirecte (g.i.) par fragmentation et différenciation du contenu protoplasmique en zoospores $(Z)$ assurera la séparation des différents génotypes et augmentera la diversité des foyers infectieux. Ainsi, l'isolement d'une zoospore devenue $\frac{\mathrm{av}_{2}}{\mathrm{av}_{2}}$, c'est-à-dire homozygote récessive, donnera naissance à un thalle, différent du thalle d'origine, qui révèlera un caractère masqué jusqu'alors, à savoir la virulence 2 (fig. 1D).

L'intervention de la reproduction sexuelle et de la méiose, qui assurent au cours du cycle de développement le brassage des informations, sont des mécanismes plus efficaces pour rendre compte de la diversité et de la variabilité intraspécifique. Ils devront être pris en considération dans nos aires de cultures puisque la différenciation des organes sexuels chez les souches de type $A_{1}$, potentiellement hermaphrodites, dépendra uniquement de la proximité d'un partenaire de type $A_{2}$ de n'importe quelle espèce du même genre (fig. 1E : induction hormonale). La présence de $P$. drechsleri (SKIDMORE et al., 1984) sur les feuilles de pomme de terre impose maintenant de considérer sérieusement cette nouvelle possibilité.

Ainsi un thalle hétérozygote $\frac{\mathrm{AV}_{2}}{\mathrm{av}_{2}}$ produit 2 sortes de gamètes $\left(\mathrm{AV}_{2}\right.$ et $\left.\mathrm{av}_{2}\right)$; lors de la caryogamie les combinaisons alléliques seront $\frac{\mathrm{AV}_{2}}{\mathrm{av} v_{2}}, \frac{\mathrm{AV}}{\mathrm{AV}}$ et $\frac{\mathrm{av}_{2}}{\mathrm{av} v_{2}}$. L'oospore qui résulte de la fécondation possédera un noyau semblable $\left(\frac{\mathrm{AV}}{\mathrm{av}_{2}}\right)$ (fig. $1 \mathrm{~F}_{1}$ ) ou différent $\left(\frac{\mathrm{AV}_{2}}{\mathrm{AV_{2 }}}\right.$ ou $\left.\frac{\mathrm{av}_{2}}{\mathrm{aV_{2 }}}\right)$ (fig. $1 F_{2}$ ou $F_{3}$ ) de ceux de la souche d'origine autofécondée (fig. 1A). Par conséquent, si la reproduction sexuelle est induite chez un thalle hétérozygote, $50 \mathrm{p}$. 100 des oospores formées maintiendront la structure hétérozygote et ne surmonteront pas la résistance $\mathbf{R}_{2}$ et $25 \mathrm{p}$. 100, devenues homozygotes récessives pour le caractère de virulence 2 , exprimeront une nouvelle potentialité : la compatibilité avec des variétés de pomme de terre porteuses du gène de résistance $R_{2}$. Cette situation augmente considérablement les chances de voir apparaître dans la nature de nouvelles races, comme nous venons de le montrer pour la race 2 . Mais ces nouvelles races pourront demeurer en sommeil très longtemps sous la forme d'oospores dormantes, dont la durée de vie atteint plusieurs années. 
L'introduction au champ de variétés de pommes de terre dotées d'un ou même de plusieurs nouveaux gènes de résistance spécifique ne garantit donc pas une protection durable.

Reçu le 10 janvier 1985.

Accepté le 21 novembre 1985.

\section{RÉFÉRENCES BIBLIOGRAPHIQUES}

Boccas B., 1971. Contribution à l'étude de la reproduction sexuelle chez Phytophthora palmivora, parasite des cultures tropicales. Thèse $3^{\mathrm{e}}$ Cycle, Orsay, $62 \mathrm{p}$.

Boccas B., 1978. La reproduction sexuée chez les Phytophthora. Ses voies et quelques-unes de ses conséquences génétiques. Thèse Doctorat Etat, Orsay, $175 \mathrm{p}$.

Denward T., 1970. Differentiation in Phytophthora infestans. II Somatic recombination in vegetative mycelium. Hereditas, 66, 35-48.

Erwin D. C., 1982. Variability within and among species of Phytophthora (68 p.). In D. C. Erwin, S. Bartnicki-Garcia \& P. H. Tsao : " Phytophthora, its biology, taxonomy, ecology and patho$\log y$ ». Am. Phytopathol. Soc., St. Paul, Minn., 392 p.

Gallegly M. E., Eichenmuller J. J., 1959. The spontaneous appearence of the potato race 4 character in cultures of Phytophthora infestans. Am. Potato J., 36, 45-51.

Gallegly M. E., Galindo J., 1958. Mating types and oospores of Phytophthora infestans in nature in Mexico. Phytopathology, 48, 274-277.

Hobe M. A., 1981. Pathogenic variability of Phytophthora megasperma $f$. $s p$. glycinea isolates from $\mathrm{N}$-W Ohio soils. M. Sci Thesis. The Ohio State Univ. Columbus, Ohio, $32 \mathrm{p}$.

Ko W. H., 1978. Heterothallic Phytophthora : evidence for hormonal regulation of sexual reproduction. J. Gen. Microbiol., 107, 15 18.

Leach S. S., Rich A. E., 1969. The possible role of parasexuality and cytoplasmic variation in race differentiation in Phytophthora infestans. Phytopathology, 59, 1360-1365.

I.e Grand-Pernot F., Pellegrin F., 1976. Nature du cycle végétatif des Phytophthora parasitica Dastur et palmivora (Butler) Butler. Ann. Phytopathol., 8, 379-388.

Le Grand-Pernot F., 1983. Tentative de domestication in vitro d'un champignon biotrophe Phytophthora infestans (Mont.) de Bary. Organisation du génome, ses conséquences. Thèse Doctorat Etat, Orsay, $210 \mathrm{p}$.
Maïa N., Venard P., Lavrut F., 1976. Etude des divisions mitotiques et méiotiques du cycle de Phytophthora capsici. Ann. Phytopathol., 8, 141-146.

Malcolmson J. F., 1970. Vegetative hybridity in Phytophthora infestans. Nature, 225, 971-972.

Mortimer A. M., Shaw D. S., Sansome E. R., 1977. Genetical studies of secondary homothallism in Phytophthora dreschsleri. Arch. Microbiol., 111, 255-259.

Sansome E. R., 1977. Polyploidy and induced gametangial formation in British isolates of Phytophthora infestans. J. Gen. Micro biol., 99, 311-316.

Satour M. M., Butler E. E., 1968. Comparative morphological and physiological studies on the progenies from intraspecific matings of Phytophthora capsici. Phytopathology, 58, 183-192.

Savage E. J., Clayton C. W., Hunter J. H., Brenneman J. A., Laviola C., Gallegy M. E., 1968. Homothallism, heterothallism and interspecific hybridization in the genus Phytophthora. Phytopatho$\log y, \mathbf{5 8}, 1004-1021$.

Shaw D. S., Janssen B., Khaki J. A., 1973. The genetics of Phytophthora drechsieri. Cah. ORSTOM. Biol., 20, 57-58.

Shepherd C. J., Pratt B. H., 1974. Temperature-growth relations and genetic diversity of $\mathrm{A}_{2}$ mating type isolates of Phytophthora cinnamomi in Australia. Aust. J. Bot., 22, 231-249.

Skidmore D. I., Shattock R. C., Shaw D. S., 1984. Oospores in cultures of Phytophthora infestans resulting from selfing induced by the presence of $P$. drechsleri isolated from blighted potato foliage. Plant Pathol., 33, 173-183.

Tooley P. W., Fry W. E., 1983. Genetic variation in Phytophthora infestans identified through isozyme analysis. Phytopathology, 73, 827.

Wilde P., 1961. Beitrag zur Kenntnis der Variabilität von Phytoph thora infestans (Mont.) de Bary. Arch. Mikrobiol., 40, 163-165. 